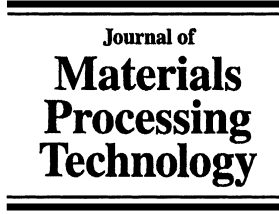

Journal of Materials Processing Technology 97 (2000) 100-106

www.elsevier.com/locate/jmatprotec

\title{
Three-dimensional numerical simulation of the deep-drawing process using solid finite elements
}

\author{
L.F. Menezes ${ }^{\mathrm{a}, *}$, C. Teodosiu ${ }^{\mathrm{b}}$ \\ ${ }^{a}$ Departamento de Engenharia Mecânica, Universidade de Coimbra, Pólo II, 3030 Coimbra, Portugal \\ ${ }^{\mathrm{b}}$ Laboratoire des Propriétés Mécaniques et Thermodynamiques des Matériaux, UPR CNRS, Université de Paris Nord, 93430 Villetaneuse, France
}

Received 1 June 1998

\begin{abstract}
The main goal of this work is to present a three-dimensional mechanical model for the numerical simulation of the deep-drawing process. The model takes into account the large elastoplastic strains and rotations that occur in the deep-drawing process. Hill's orthotropic yield criteria with isotropic and kinematics hardening describes the anisotropic plastic properties of the sheet. Coulomb's classical law models the frictional contact problem treated with an augmented Lagrangian approach. This method yields a mixed system where the final unknowns of the problem are static (frictional contact forces) and kinematic (displacements) variables. To solve this problem use is made of a fully implicit algorithm of Newton-Raphson type. Three-dimensional isoparametric finite elements with a selective reduced integration are used for the spatial discretization of the deformed body. The geometry of the forming tools is modelled by Bézier surfaces. The numerical results of the deep-drawing of a square cup are presented to focus their good agreement with the results of experiment. (C) 2000 Elsevier Science S.A. All rights reserved.
\end{abstract}

Keywords: Deep-drawing; Numerical simulation; Finite elements

\section{Introduction}

The deep-drawing process is used widely in a variety of industrial areas. In all its years of use, the industrial experience gained has always been used for the conception of new parts. In reality, conception cycles of shorter periods have significantly cut down the time available for the optimisation of the forming tools. This optimisation process requires a good understanding of the technological process and the parameters involved, such as the lubrification conditions, the forces over the forming tools, the gap between punch and die, etc. The use of numerical simulation could contribute towards the development and optimisation of the process, leading to significant economic and technical gains.

The application of the finite element method to the numerical simulation of the deep-drawing process has evolved in a significant way in the course of the last few years. Many of the problems associated with numerical simulation of this process have been solved or at least are better understood, of which the following are noteworthy;

*Corresponding author. Tel.: +351-39790700; fax: +351-39790701 E-mail address: luis.menezes@mail.dem.uc.pt (L.F. Menezes) the correct model of the phenomena at play, and the development of sound numerical methods capable of coping with the strong non-linearity generally associated with it. Further to this, thanks to the calculation speed currently available, it has been possible to perform three-dimensional simulations that account for the increasing industrial interest in this field. Therefore, in the present paper certain general considerations have been made of the three-dimensional mechanical model and the numerical methods used in the algorithms of the program DD3IMP ${ }^{1}$, developed in order to simulate the industrial processes of deep-drawing.

\section{Mechanical model}

Deep-drawing process modelling puts forward complex problems due to the non-linearity of the behaviour laws, to the presence of elastoplastic transformations and to the nonconstant boundary conditions of contact with friction. A mechanical model used in numerical simulation should take all of these aspects into account, requiring careful simpli-

\footnotetext{
${ }^{1}$ DD3IMP - contraction of "Deep Drawing 3D IMPlicit finite element
} code". 
fications in order not to affect the precision of the results. In the present case, the model considers the problem as a process of large elastoplastic transformations. To correctly evaluate the shear and bending effects, no kinematic simplification is made of the membrane or shell type. The plastic behaviour of the material is described by Hill's criteria with isotropic and kinematic work-hardening, and by an associated flow rule. The Coulombs law describes the contact with friction and its evolution is controlled by a mixed formulation, using an augmented Lagrangian approach. The model establishes the contact between a deformed body (sheet) and a rigid body (forming tools). The kinematic description uses an updated Lagrangian approach. It is also taken into account that the elastic part of the transformation leads to small strains when compared to the unit strain, although big rotations could occur, thus making the hypothesis of small elastic strains applicable.

\subsection{Basic equations of elastoplastic formulation}

Under the aforementioned conditions, it is possible to deduce the differential form of the elastoplastic behaviour law, the general form of which is as follows [1]:

$\dot{\boldsymbol{\sigma}}^{J}=\boldsymbol{C}^{\mathrm{ep}}: \boldsymbol{D}$,

where $\dot{\boldsymbol{\sigma}}^{J}$ is the rate of variation according to Jaumann of the Cauchy stress tensor $\boldsymbol{\sigma}$, which is given by:

$\dot{\boldsymbol{\sigma}}^{J}=\dot{\boldsymbol{\sigma}}+\boldsymbol{\sigma} \boldsymbol{W}-\boldsymbol{W} \boldsymbol{\sigma}$,

where $\dot{\boldsymbol{\sigma}}$ stands for the time derivative of the Cauchy stress tensor and $\boldsymbol{W}$ is the total spin tensor defined by:

$\boldsymbol{W}=\dot{\boldsymbol{R}} \boldsymbol{R}^{T}$,

in which $\boldsymbol{R}$ is the orthogonal elastic rotation tensor. In Eq. (1) $\boldsymbol{D}$ is the strain rate tensor and $\boldsymbol{C}^{\mathrm{ep}}$ is the elastoplastic module, the calculation of which depends on the algorithms used for the time integration of the behaviour law. The strain rate is decomposed into elastic and plastic parts, $\boldsymbol{D}^{\mathrm{e}}$ and $\boldsymbol{D}^{\mathrm{p}}$, respectively. $D^{\mathrm{e}}$ is given by the Jaumann objective derivative of the strain tensor $\boldsymbol{\varepsilon}$ and $\boldsymbol{D}^{\mathrm{p}}$ is a deviatoric tensor, given by the associated inviscid flow rule:

$\boldsymbol{D}^{p}=\dot{\lambda} \frac{\partial f(\bar{\sigma}, Y)}{\partial \boldsymbol{\sigma}}$,

in which $\dot{\lambda}$ is the plastic multiplier that can be calculated after the condition $\dot{f}=0$, where $f$ is the plastic potential which is identified as the scalar function that defines the elastic limit surface, described by the general quadratic yield condition:

$f(\bar{\sigma}, Y)=\bar{\sigma}-Y=0$.

$Y$ denotes the flow stress in simple traction. Its evolution depends on the isotropic work hardening modelled by the Swift law:

$Y=C\left(\varepsilon_{0}+\bar{\varepsilon}^{\mathrm{p}}\right)^{n}$, where $C, \epsilon_{0}$ and $n$ are material parameters. $\bar{\sigma}$ is the equivalent stress defined by the quadratic equation:

$\bar{\sigma}^{2}=(\boldsymbol{\sigma}-\boldsymbol{X}): \boldsymbol{M}:(\boldsymbol{\sigma}-\boldsymbol{X})$

$\boldsymbol{M}$ is a fourth-order symmetric anisotropy tensor, whilst $\boldsymbol{X}$ is a second-order tensor, symmetric and deviator, called the back-stress tensor, which is associated with the kinematic work hardening, and is given by the Prager's law:

$\dot{X}^{J}=k D^{\mathrm{p}}$

where $k$ is a material parameter and $\dot{X}^{J}$ is the Jaumann derivative of $\boldsymbol{X}$.

In order to account for the evolution of $\boldsymbol{M}$ during the deformation process, it is supposed that the material, initially orthotropic, remains so during the deformation. The orthotropic axis are subjected to the time-dependent rotation $\boldsymbol{R}$ whose evolution is governed by Eq. (3). Therefore, the evolution of $\boldsymbol{M}$ is given as a function of the initial anisotropy tensor $\boldsymbol{M}$, corresponding to the Hill's quadratic yield condition, by the equation:

$\boldsymbol{M}=\boldsymbol{R}\left(\boldsymbol{R} \boldsymbol{M} \boldsymbol{R}^{T}\right) \boldsymbol{R}^{T}$.

\subsection{Principle of virtual velocities}

In the updated Lagrangian scheme, the configuration of the sheet at time $t$ is taken as the reference configuration for the time interval $[t, t+\Delta t]$. At the end of the increment $\Delta t$, the configuration is updated and taken as the reference configuration for the next increment. Assuming that a deformed body, submitted to external forces, has a domain $\Omega_{0}$ with surface $\Sigma_{0}$ at instant $t$, the principle of virtual velocities written in the reference configuration is given by the following equation [1]:

$\int_{\Omega_{0}} \boldsymbol{S}: \frac{\partial \boldsymbol{\delta} \boldsymbol{v}}{\partial \boldsymbol{x}_{\boldsymbol{0}}} \mathrm{d} \Omega=\int_{\Sigma_{0}} \boldsymbol{s}^{*} \cdot \delta \boldsymbol{v} \mathrm{d} \Sigma$

which is valid for any virtual velocity field $\delta \boldsymbol{v} . \boldsymbol{s}^{*}$ stands for the prescribed nominal stress vector expressing the external load, $x_{0}$ is the vector position of a generic material point at time $t$ and $S$ is the first Piola-Kirchhof stress tensor.

The behaviour law defined by Eq. (1) is formulated in velocities. However, the principle of virtual velocities in Eq. (10) involves the total stresses. Following this, it is necessary to linearize the principle in order to involve the tensors that occur directly in the formulation of the constitutive laws.

\subsection{Unilateral contact with friction}

The numerical simulation of the deep-drawing process requires a good prediction of the evolution of the contact conditions between the forming tools and the metal sheet to be formed. The main difficulty is that the boundary conditions are time-dependent. For this reason, a classical treatment of them, particularly in the case of using a fully 
implicit numerical method, is virtually impossible. The inequations associated with contact with friction contribute towards a strong non-linearity of the models, necessitating the use of fairly strong numerical methods for the solving of the global problem. The augmented Lagrangian approach is currently quite popular for the solving of contact friction problems. In the context of the finite element method, this approach has been applied successfully [2-4], presenting a significant development. Heege [5] generalised the use of the augmented Lagrangian approach for contact between a deformed body and a rigid obstacle of general shape. This generalisation was applied successfully in the simulation of the deep-drawing process [1]. This method subscribes to a mixed formulation in which the kinematic variables (displacements) and static variables (frictional contact forces) are the final unknowns of the problem. This leads to a nonlinear system that can be solved by a method of the NewtonRaphson type.

Eq. (10) expresses the conditions necessary for a structural balance in the absence of contact and friction and is equivalent to the following generic form:

$\boldsymbol{F}^{\text {int }} \boldsymbol{u}-\boldsymbol{F}^{\mathrm{ext}}=0$,

where $\boldsymbol{F}^{\text {int }}(\boldsymbol{u})$ and $\boldsymbol{F}^{\text {ext }}$ are the vectors of internal and external forces and $\boldsymbol{u}$ is the displacement field, which is the vector solution of the problem. With the inclusion of contact with friction, Eq. (11) remains valid although constrained by the boundary conditions associated with the contact with friction problem. An augmented multiplier is therefore introduced, $\lambda_{\mathrm{n}}+r d_{\mathrm{n}}(u)$, where $r$ is the penalty coefficient, $\lambda_{\mathrm{n}}$ is the normal frictional force on the surface at the contact point and $d_{\mathrm{n}}$ is the contact normal distance. It is possible in this way to formulate an augmented Lagrangian $L^{r}(\boldsymbol{v}, \boldsymbol{\gamma})$, of virtual variables $\boldsymbol{v}$ and $\boldsymbol{\gamma}$ and of parameters $\boldsymbol{u}$ and $\lambda$, giving rise to an exactly equivalent problem for all strictly positive values of the penalty coefficient $r$, and which can generally be written as follows [1,2]:

$\left[\frac{\partial}{\partial \boldsymbol{v}}, \frac{\partial}{\partial \gamma}\right] \boldsymbol{L}^{r}(\boldsymbol{u}, \boldsymbol{\lambda})=\left\{\begin{array}{l}\boldsymbol{F}^{\mathrm{int}} \boldsymbol{u}+\boldsymbol{F}^{\mathrm{equi}}(\boldsymbol{u}, \boldsymbol{\lambda})-\boldsymbol{F}^{\mathrm{ext}}=0 \\ \boldsymbol{F}^{\mathrm{sup}}(\boldsymbol{u}, \boldsymbol{\lambda})=0\end{array}\right.$

Therefore, $\boldsymbol{F}^{\text {ext }}$ represents the classical external forces, $\boldsymbol{F}^{\text {equi }}(\boldsymbol{u}, \boldsymbol{\lambda})$ imposes the boundary conditions originating from the contact with friction and $\boldsymbol{F}^{\text {sup }}(\boldsymbol{u}, \boldsymbol{\lambda})$ takes into account the supplementary terms necessary for the control of the frictional contact boundary conditions [1]. The problem is therefore differentiable and without constraints, making possible the use of a method of the Newton-Raphson type for its resolution.

From a mathematical point of view, Eq. (12) represents a system of six equations for six unknowns, for each point of the deformed body. These unknowns are three displacements $(\boldsymbol{u})$ and three contact forces $(\boldsymbol{\lambda})$, which justifies the designation of mixed formulation in $(\boldsymbol{u}, \boldsymbol{\lambda})$.

The use of computer codes in industrial applications requires the adaptation of the contact algorithms to take into account forming tools described by parametric surfaces (Bézier, splines,...). As a result, it is no longer possible to analytically calculate some contact parameters such as the normal distance, the normal vector to the surface, the relative displacements between deformed body and forming tools, etc. Therefore, it is necessary to introduce numerical algorithms that can lead to a minute and astute management with regard to contact detection and the calculation of the contact parameters over this type of surface [1].

\section{Numerical methods}

The use of an implicit method for the simulation of the deep-drawing process ensures the structural balance at any given instant of the calculation. Taking the known configuration at instant $t$, the equilibrium equations are solved for the instant $t+\Delta t$. The static structural balance is assured by an iterative scheme in the vicinity of the arrived configuration at instant $t+\Delta t$. The algorithms are much more sophisticated than in the explicit method, allowing greater increments. However, it has some disadvantages when compared to the explicit method. In fact, implicit algorithms have no protection against eventual numerical instability, and divergence can occur during the iteration process. Generally, this is associated with severe changes in the boundary conditions or in the strain evolution during an increment. It is therefore necessary to alter the algorithms favourably in a generally complex manner so that these difficulties can be sorted out without affecting the quality of the problem solution [1].

Two types of non-linearities occur in the numerical simulation of the deep-drawing process. One of them concerns the large elastoplastic transformations whilst the other is with respect to the contact with friction. These are two interrelated issues: however, most existing calculation programmes reserve an iterative loop for the solving of each of these non-linearities [6-9]. The mixed formulation described earlier allows the transformation of the non-differentiability of both the contact and frictional laws to a partial differentiable non-linear problem, making the solution possible by a method of the Newton-Raphson type. Therefore, only an iterative loop strategy can be adopted [1].

\subsection{The implicit method}

In the implicit method, equilibrium equations and the coherence condition are imposed on the configuration at the instant $t+\Delta t$, which is unknown. Therefore, this configuration has to be determined by successive approximations. By an explicit method, for example, an approximate first solution for the incremental displacements, stresses and frictional contact forces can be calculated. Generally, this solution satisfies neither the variational principle nor the coherence condition, due to the non-linearity of the behaviour law and to the kinematics of the process that is being 
simulated. Therefore, the correction of the solution lies in the iterative Newton-Raphson method [1]. Consider that at an instant $t$, corresponding to a configuration $C^{[N-1]}$, the stress state of the deformed body satisfies the principle of virtual velocities as well as the behaviour law. After calculating the explicit solution of the increment $[t, t+\Delta t]$, the actualised configuration $C^{[N, 0]}$ is given as a function of the incremental displacements and frictional contact forces, $\Delta \boldsymbol{u}^{[0]}$ and $\Delta \lambda^{[0]}$, by:

$\boldsymbol{x}^{[N, 0]}=\boldsymbol{x}^{[N-1]}+\Delta \boldsymbol{u}^{[0]}, \quad \lambda^{[N, 0]}=\boldsymbol{\lambda}^{[N-1]}+\Delta \boldsymbol{\lambda}^{[0]}$.

With this solution, it is possible to calculate the incremental strains and rotations and to integrate the behaviour law in order to determine the stress field and the plastic strains at the instant $t+\Delta t$ [1]. This is the first approximation of the configuration and of the state of the deformed body at the instant $t+\Delta t$. To calculate the incremental displacements and the corrected incremental frictional contact forces, Eq. (12) is linearized with respect to the displacements in the neighbourhood of the configuration $C^{[N, 0]}$, thus arriving at a linear system of equations of unknowns $\mathrm{d} \boldsymbol{u}^{[1]}$ and $\mathrm{d} \lambda^{[1]}$. After the resolution of this system:

$\boldsymbol{x}^{[N, 1]}=\boldsymbol{x}^{[N-1]}+\Delta \boldsymbol{u}^{[1]}, \quad \lambda^{[N, 1]}=\lambda^{[N-1]}+\Delta \lambda^{[1]}$,

where,

$\Delta \boldsymbol{u}^{[1]}=\Delta \boldsymbol{u}^{[0]}+d \boldsymbol{u}^{[1]}, \quad \Delta \boldsymbol{\lambda}^{[1]}=\Delta \boldsymbol{\lambda}^{[0]}+d \boldsymbol{\lambda}^{[1]}$.

This iterative process is repeated until the configuration obtained is satisfactory, i.e., until the iteration when the outof-balance forces attain a lower value to the imposed limit. Then, the configuration at the end of the increment is considered calculated, passing on therefore to the next increment.

Quadratic convergence in the vicinity of the solution is one of the main advantages of this method. On the other hand, numerical instabilities are frequent. The difficulty of the numerical treatment is due to the fact that the problems of contact introduce inequalities that lead to strong nonlinearities. The behaviour law associated with the deformed body also contributes towards the strong non-linearity of the problem to be solved. The quadratic convergence area in the vicinity of the solution is necessarily included in a differentiable region characterised by the "correct" contact status of each point of the surface of the deformed body (no contact, stick contact or slip contact). During the first iterations, the algorithm should determine the correct contact status. In this way, conclusions on the existence of the quadratic convergence can be made, but only after the moment when no more changes in the contact status occur [1]. Numerical tests have shown that the global convergence depends mainly on the following factors: the penalty coefficient $r$, the behaviour law of the deformed body, the friction coefficient, the tool geometry, the finite element mesh of the deformed body and the solution at the beginning of the iteration process. Although the convergence behaviour is a function of these parameters, in the case of divergence it is extremely difficult to identify the parameters responsible. Further to this, when solving a real problem, the behaviour law, and the friction coefficient, as well the tool geometry, are unchangeable factors as they depend on the type of problem to be solved. In case divergence does occur, the way to avert it is to act on the value of $r$, on the finite element mesh and on the solution at the beginning of the iterative loop. The results of the numerical tests undertaken show that it is always possible to overcome the problems of convergence by modifying the cited parameters, without a loss in the quality of the results, which show the method developed to be highly sound and reliable.

\section{Finite elements}

The use of solid finite elements considers the sheet as a three-dimensional domain, which is the more realistic way to model the process. The simultaneous contact on both sides of the sheet is naturally solved without any particular strategy. Further to this, with these elements it is possible to calculate accurately the stress gradients over the thickness of the sheet as well the evolution of the sheet thickness during the simulation. However, its use requires certain care, which is trying in terms of the CPU time required: At least two thick layers of elements should be used in order to accommodate the stress gradients that occur in this direction and the ratio between the dimensions of the elements on the sheet plane and over the thickness should be small to avoid deterioration of the matrix of the linear system being solved.

In order to choose a finite element to be used in the simulation, one should take into account aspects such as the plastic incompressibility, the geometry of the operation, its behaviour in bending and shear, the contact with friction and the cost in terms of time of CPU. In the present work, an isoparametric, hexahedral element is used, associated with a selective reduced integration technique $[10,11]$. This element has eight nodes, eight Gauss points for complete integration and one for reduced integration. It uses the same interpolation functions to interpolate the spatial co-ordinates $\boldsymbol{x}$ and the incremental displacements $\Delta \boldsymbol{u}$ in its domain. There are then the following relations:

$\boldsymbol{x}=\sum_{a=1}^{n_{\mathrm{en}}} N_{a}^{\mathrm{e}}(\xi, \eta, \zeta) \boldsymbol{x}_{a}^{\mathrm{e}}, \quad \Delta \boldsymbol{u}=\sum_{a=1}^{n_{\mathrm{en}}} N_{a}^{\mathrm{e}}(\xi, \eta, \zeta) \Delta \boldsymbol{u}_{a}^{\mathrm{e}}$

where $\Delta \boldsymbol{u}_{a}^{\mathrm{e}}$ and $\boldsymbol{x}_{a}^{\mathrm{e}}$ are the incremental displacement and position vectors of the node $a$ of the element $e ; n_{\mathrm{en}}$ is the number of nodes of the element; $\xi, \eta$ and $\zeta$ are the canonical co-ordinates; and $N_{a}^{\mathrm{e}}$ is the interpolation function associated with node $a$.

The isoparametric elements have a deficient behaviour when used to solve elastoplastic problems [12]. In fact, using a complete integration scheme increases the stiffness of the element causing the appearance of artificial hydrostatic 

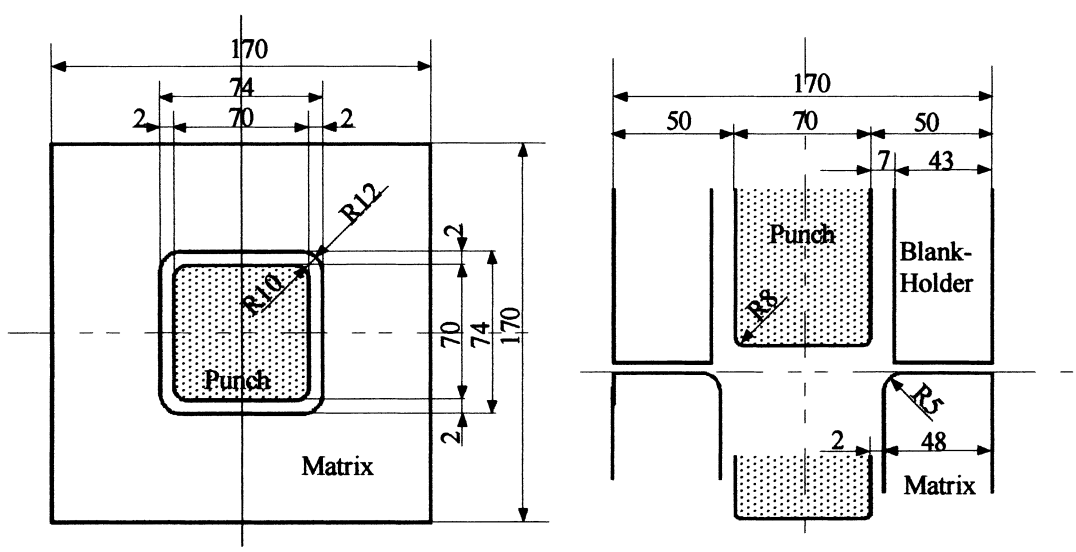

Fig. 1. Forming tools for the deep-drawing of a square cup.(dimensions:mm).

stresses, which leads to a complete deterioration of the solution. This phenomenon is associated with the isochoric nature of plastic deformation. Thus, the distortions that can occur in the volume conservation are compensated for by a volume deformation of elastic origin. The effect can be eliminated by the use of a selective reduced integration method ("assumed strain methods") [10,13-15]. Here, a reduced integration is used only in particular terms of the stiffness matrix. Regarding the method used in the present work, the hydrostatic components of the velocity and virtual velocity gradients are both considered constant in the whole element and are calculated at its central point (point of reduced integration) [10]. This method is equivalent to the application of a complete integration where the velocity gradient field $\boldsymbol{L}$ and the virtual velocity gradient field $\delta \boldsymbol{L}$ is calculated as follows:

$L_{k l}=N_{b, l} v_{b k}+a_{b q} v_{b q} \delta_{k l}, \quad \delta L_{i j}=N_{a, j} \delta v_{a i}+a_{a p} \delta v_{a p} \delta_{i j}$

where $i, j, k, l, p$ and $q$ note the spatial co-ordinate indices and:

$a_{a p}=(1 / 3)\left(\bar{N}_{a, p}-N_{a, p}\right)$,

where $\bar{N}_{a, p}$ is the value of $N_{a, p}$ at the central point of the element.

By the spatial discretization of the deformed body with finite elements, and applying the usual assembling technique to the elemental matrices, a global linear system is obtained as follows [1]:

$\left\{\begin{array}{c}{\left[K_{A B}\right]_{i j}\left\{\mathrm{~d} u_{B}\right\}_{j}+\left[A_{A}\right]_{i j}\left\{\mathrm{~d} u_{A}\right\}_{j}+\left[C_{A}\right]_{i j}\left\{\mathrm{~d} \lambda_{A}\right\}_{j}=\left\{f_{A}^{1}\right\}_{i},} \\ \times\left[A_{A}\right]_{i j}\left\{\mathrm{~d} u_{A}\right\}_{j}+\left[B_{A}\right]_{i j}\left\{\mathrm{~d} \lambda_{A}\right\}_{j}=\left\{f_{A}^{2}\right\}_{i} .\end{array}\right.$

where $\mathrm{d} u$ and $\mathrm{d} \lambda$ correspond to the increase in displacement and the frictional contact force, respectively. $A$ and $B$ correspond to the global numeration of the nodes. The term $\left[K_{A B}\right]_{i j}$ is the traditional global stiffness matrix and is independent of the contact status of the nodes. The remaining matrices will depend on the contact status of node $A$ and are associated with the contact operators $\boldsymbol{F}^{\text {equi }}$ and $\boldsymbol{F}^{\text {sup }}$.

\section{Numerical example}

This section shows some results of the numerical simulation of the deep-drawing of a square cup. The dimensions of the forming tools are described in Fig. 1.

Due to geometrical symmetry, only one quarter of the global structure is simulated. The initial dimensions of the metal sheet are $150 \times 150 \times 0.81 \mathrm{~mm}$ and it has the following mechanical properties: $E=0.71 \times 10^{5} \mathrm{MPa} ; \nu=0.33$; the Swift law: $\bar{\sigma}=576.79 \times\left(0.01658+\bar{\varepsilon}^{p}\right)^{0.3593} \mathrm{MPa}$; the Hill coefficients: $F=0.5932, G=0.5848, H=0.4152$, $L=M=N=1.272$. Further, a friction coefficient of 0.162 and a blank-holder force of $4900 \mathrm{~N}$ were used. In order to describe the forming tools, nine Bézier surfaces were used for the matrix, nine for the punch and three for the blank-holder. The sheet was discretized in a regular mesh composed of 1250 hexahedrons, with two element layers in the thickness direction.

Fig. 2 compares the numerical and experimental [16] results of the distribution of the thickness strains along the $O x$ axis (Fig. 1), after a punch travel of $15 \mathrm{~mm}$. The numerical results are in good agreement with the experimental results.

Fig. 3 shows the isovalues of the thickness strains after a punch travel of $20 \mathrm{~mm}$. As can be observed, the strain attains

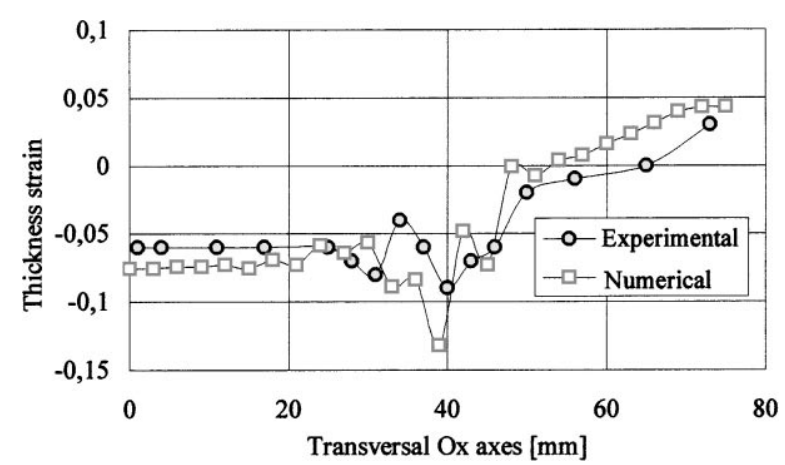

Fig. 2. Distribution of the thickness strain along the $O x$ axis (Fig. 1), after a punch travel of $15 \mathrm{~mm}$. 


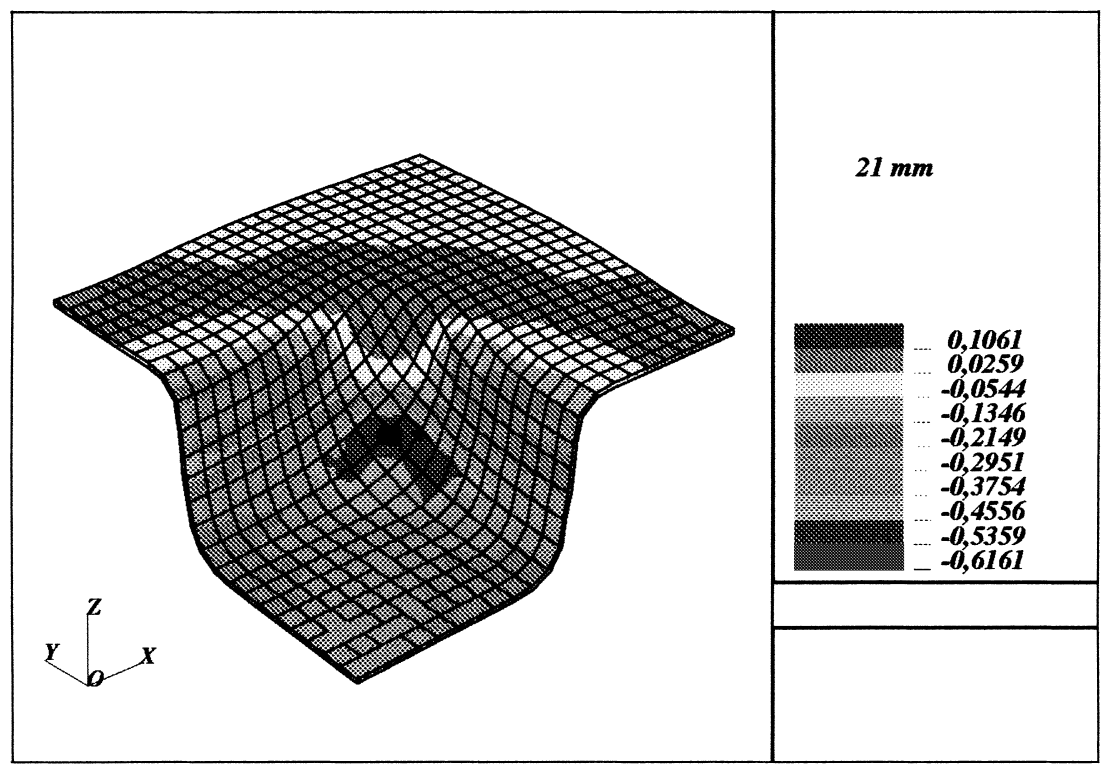

Fig. 3. Isovalues of the thickness strain after a punch travel of $21 \mathrm{~mm}$.

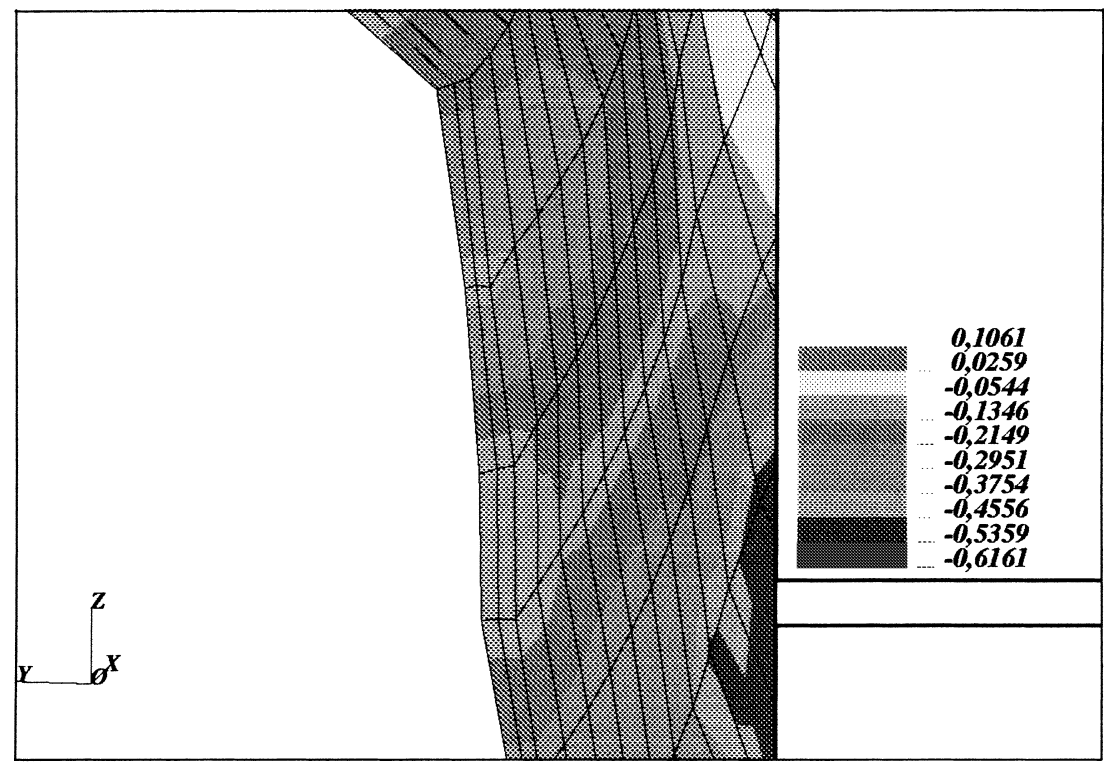

Fig. 4. Isovalues of the thickness strain after a punch travel of $21 \mathrm{~mm}$ (detail in the sheet vertical wall).

values of $60 \%$, which for the present case is too high and hinder the success of the deep-drawing process. In fact, the experimental results present a rupture in the sheet after a punch travel of around $20 \mathrm{~mm}$ [16]. Fig. 4 presents a detail of the thickness strains on the vertical wall of the cup. A local decrease of the sheet thickness can be observed that could mean a strain localisation, confirming the failure of the operation.

The deep-drawing of the square cup allows comparison with the numerical results obtained through the DD3IMP code with an example of real industrial interest. Despite the results being consistent, some systematic differences were observed in relation to the experimental results which can be reduced by using a more refined finite element mesh. This example enhances the use of solid finite elements. In fact, the experimental failure of the process was well predicted in the numerical simulation.

\section{Conclusions}

This paper presents a summary of a mechanical model for the numerical simulation of the deep-drawing process that is keeping with the development of the DD3IMP code. Special 
attention has been given to the use of the elastoplastic behaviour law and to the use of an augmented Lagrangian approach in order to include the frictional contact problem. The resultant problem is solved by an implicit method with one single iterative loop. Three-dimensional isoparametric finite elements are used for the spatial discretization of the deformed body. The forming tools are modelled by Bézier surfaces enabling a direct link with CAD codes.

The numerical results obtained in the simulation of the deep-drawing of a square cup were compared with experimental results. Despite a relatively low number of finite elements used in this simulation, the numerical and experimental results were found to be in agreement.

\section{Acknowledgements}

First author is indebted to JNICT for financial support through FEDER and Programa PRAXIS XXI.

\section{References}

[1] L.F. Menezes, Modelação tridimensional e simulação numérica dos processos de enformação por deformação plástica, aplicação à estampagem de chapas metálicas, Ph.D Thesis, Coimbra, 1994.

[2] P. Alart, A. Curnier, A mixed formulation for frictional contact problems prone to Newton like solution methods, Comp. Meth. Appl. Mech. Eng. 92(3) (1991) 353.

[3] G. Saxe, Z.Q. Feng, New inequality and functional for contact with friction: the implicit standard material approach, Mech. Struct. and Mach. 19(3) (1991) 301.

[4] J.C. Simo, T.A. Laursen, An augmented Lagrangian treatment of contact problems involving friction, Comp. Str. 42 (1992) 97.

[5] A. Heege, Simulation numérique 3D du contact avec frottement et application à la mise en forme, Thèse de doctorat, INP-Grenoble, 1992.
[6] F. Horkay, M. Jean, F. Mehrez, Unilateral contact and dry friction in numerical simulation of deep drawing, Numerical Methods in Industrial Forming Processes, NUMIFORM'89, Balkema, Fort Collins, Colorado, 1989.

[7] M. Jean, G. Touzot, Implementation of unilateral contact and dry friction in computer codes dealing with large strain stress elastoplastic problems, Journal de Mécanique Théorique et Appliquée, (special issue) Numerical Methods in Mechanics of Contact Involving Friction, 1988, p. 145.

[8] M. Raous, P. Chabrand, F. Lebon, Numerical methods for frictional contact problems and applications, Journal de Mécanique Théorique et Appliquée, (special issue), Numerical Methods in Mechanics of Contact Involving Friction, 1988, p. 111.

[9] C. Teodosiu, H.L. Cao, T. Ladreyt, J.M. Detraux, Implicit versus explicit methods in the simulation of sheet metal forming, FEsimulation of 3D sheet metal forming processes in automotive industry, VDI Berichte Nr. 894, Zurich, 1991, p. 601.

[10] T.J.R. Hughes, Generalization of selective reduced integration procedures to anisotropic and nonlinear media, Int. J. Num. Meth. Eng. 15 (1980) 1413.

[11] L.F. Menezes, C. Teodosiu, A. Makinouchi, 3D solid elasto-plastic elements for simulating sheet metal forming processes by the finite element method, FE-simulation of 3D sheet metal forming processes in automotive industry, VDI Berichte Nr.894, Zurich, 1991, p. 381.

[12] J.C. Nagtegaal, D.M. Parks, J.R. Rice, On numerically accurate finite element solutions in the fully plastic range, Comp. Meth. Appl. Mech. Eng. 4 (1974) 153.

[13] K.J. Bathe, E.N. Dvorkin, A four-node plate bending element based on Mindlin/Reissner plate theory and a mixed interpolation, Int. J. Num. Meth. Eng. 21 (1985) 367

[14] D.S. Malkus, T.J.R. Hughes, Mixed finite element methods reduced and selective integration techniques: a unification of concepts, Comp. Meth. Appl. Mech. Eng. 15 (1978) 63.

[15] H. Shimodaira, Equivalence between mixed models and displacement models using reduced integration, Int. J. Num. Meth. Eng. 21 (1985) 89.

[16] T. Furuhatha, Experimental results in the square cup deep-drawing, in: A. Makinouchi, E. Nakamachi, E. Onate, R.H. Wagoner (Eds.), Second Int. Conf.: Numerical simulations of 3D sheet metal forming processes - Verification of simulation with experimental, Isehara, Japan, 1993, p. 444. 